\title{
Investigation of a novel silicon-on-insulator Rib-Slot photonic sensor based on the vernier effect and operating at $3.8 \mu \mathrm{m}$
}

B. Troia

V. M. N. Passaro

passaro@deemail.poliba.it
Photonics Research Group, Dipartimento di Ingegneria Elettrica e dell'Informazione, Politecnico di Bari, via E. Orabona 4, 70125 Bari, Italy

Photonics Research Group, Dipartimento di Ingegneria Elettrica e dell'Informazione, Politecnico di Bari, via E. Orabona 4, 70125 Bari, Italy

In this paper, we present the theoretical investigation of photonic sensors based on Vernier effect with two cascade-coupled ring resonators in silicon on insulator technology. The photonic chip utilizes rib and slot waveguides designed to operate at $3.8 \mu \mathrm{m}$ mid infrared wavelength, where a number of harmful gases, chemical and biochemical analytes are spectroscopically accessible. A rigorous algorithmic procedure has been implemented for the design of such devices and novel technological solutions have been proposed according to very recent experimental results. The rib-slot sensor architecture can exhibit wavelength sensitivities as high as $20.6 \mu \mathrm{m} / \mathrm{RIU}$ and limits of detection for homogeneous sensing as low as $3.675 \times 10^{-4}$ RIU.

[DOI: http://dx.doi.org/10.2971/jeos.2014.14005]

Keywords: Homogeneous sensing, ring resonators, silicon photonics, Vernier effect

\section{INTRODUCTION}

Nowadays, photonic sensors represent a very attractive alternative for the detection of harmful gases (e.g., methane $\left(\mathrm{CH}_{4}\right)$, ethane $\left(\mathrm{C}_{2} \mathrm{H}_{6}\right)$, carbon dioxide $\left(\mathrm{CO}_{2}\right)$, sulfure dioxide $\left.\left(\mathrm{SO}_{2}\right)\right)$ and chemical and biochemical analytes, such as DNA, glucose, proteins, bacteria $[1,2]$. The increasing interest in photonic sensors is mainly justified by their ultra-high performances and simple fabrication through standard technological processes, typical in Microelectronics. In fact, limits of detection (LOD) as low as a few parts per billion (ppb) have been demonstrated for gas sensing [3,4] and minimum detectable amount of proteins as low as few femtograms (fg) have been also experimentally detected $[5,6]$.

In this context, silicon-on-insulator (SOI) photonic sensors based on resonant microcavities represent an ultra-high performance sensing platform, since it is possible to improve the light-analyte interaction being the optical signal able to propagate into the resonant cavity along multiple roundtrips, thus enhancing the chemical-to-optical signal transduction [2, 7]. In addition, the SOI technology platform is CMOS-compatible and allows the integration of photonic devices with microfluidic systems as well as low cost and large scale fabrication.

The design of photonic architectures based on multiple ring resonators (RRs) represents a possible approach for achieving some particular spectral behaviors or device operations. In this context, the well-known Vernier linear effect can be obtained by cascading two series-coupled RRs. In particular, this effect has been used in optical signal processing for the design of optical filters [8, 9]. However, the operation of such architectures has revealed very intriguing advantages and improvements in photonic sensing applications if compared to other photonic sensing platforms. For instance, a digital optical sensor based on cascaded high- $Q$ RRs with ultra high sensitivity of about $2.91 \times 10^{5} \mathrm{~nm} / \mathrm{RIU}$ (refractive index unit) and LOD as low as $10^{-6}$ RIU has been experimentally demonstrated [10]. Furthermore, a sensitivity of $2169 \mathrm{~nm} / \mathrm{RIU}$ has been also achieved by measuring the flow between deionized water and aqueous solutions of $\mathrm{NaCl}$ with different concentrations [11]. Moreover, salt solutions with different concentrations in water have been detected by using a cascaded-ring sensor with suspended Si-nanowire in SOI technology, revealing a sensitivity of $4.6 \times 10^{5} \mathrm{~nm} / \mathrm{RIU}$ and LOD of $4.8 \times 10^{-6}$ RIU $[12,13]$. Further experimental results have been recently demonstrated by cascading two Vernier modules based on SOI cascade-coupled RRs with the aim of creating a novel sensing platform for multiple and parallel detection [14], revealing a sensitivity as high as $24,300 \mathrm{~nm} /$ RIU for wavelength interrogation and $2430 \mathrm{~dB} / \mathrm{RIU}$ for intensity interrogation.

All these sensing architectures represent the state of the art of photonic sensors based on the Vernier effect and can operate in near infrared (NIR) around the operative wavelength $\lambda_{o p}=1550 \mathrm{~nm}$. Nowadays, the extension of the operation of such devices in the unexplored mid infrared (MIR) wavelength region represents a very intriguing challenge since a lot of gaseous species (e.g., methane, ethane, formaldehyde, ammonia) as well as chemical and biochemical analytes are spectroscopically accessible within this range. In addition, photonic sensing principles such as optical absorption can be also employed simultaneously with conventional homogeneous and surface sensing, resulting in a more efficient silicon photonic spectroscopic platform, able to trace the signature of the 
molecule or gas to be detected with ultra-high sensitivities $[15,16]$.

In this paper, we present the theoretical investigation of a novel photonic sensor architecture based on two cascade-coupled RRs and operating in MIR, at wavelength $\lambda_{o p}=3800 \mathrm{~nm}$. In Section 2, we present the design of the Vernier architecture underlining design criteria and technological solutions towards the device fabrication. Moreover, the theoretical investigation of silicon rib and slot waveguides employed for the design of both RRs constituting the whole photonic chip is reported. Consequently, in Section 3 simulation results of the device performance tested for homogeneous sensing are shown. Finally, Section 4 summarizes the conclusions.

\section{INVESTIGATION OF VERNIER SENSING RIB-SLOT ARCHITECTURE IN MID INFRARED}

In recent years, several research efforts have been done for extending the operation of silicon Photonics from NIR to MIR wavelength region [17]-[20]. In particular, silicon rib waveguides, silicon-on-porous silicon (Si-on-PSi) waveguides, slot waveguides as well as Mach-Zendher interferometers, multimode intereference (MMI) devices, gratings and spectrometers in SOI technology platform, have been fabricated and experimentally characterized at MIR wavelengths (e.g., $3.39 \mu \mathrm{m}$ and $3.8 \mu \mathrm{m}$ ), revealing low propagation losses and high performance operations $[17,18]$. In this context, the design of photonic sensors based on the Vernier effect is very interesting to be theoretically investigated towards future fabrication and characterization.

The proposed architecture is constituted by two cascadecoupled RRs, as sketched in Figure 1. The first RR acts as a filter and receives the optical signal from the input bus. Consequently, the signal is coupled to the second RR (i.e., the sensing RR) by means of silicon waveguide directional couplers, shown in the schematic with their corresponding power coupling coefficients $k_{i}$. The whole architecture is assumed to be covered by a silicon dioxide $\left(\mathrm{SiO}_{2}\right)$ layer, except for a window opened on the sensing RR which defines the sensible area of the Vernier architecture, where the gas or analyte to be detected can be properly concentrated. The optical signal propagating in the sensing RR is then coupled to the output bus to be processed by the external optical readout.

The operation of the Vernier sensor can be analyzed by considering the transmittance $T_{\text {tot }}$ of the cascaded configuration sketched in Figure 1. In fact, the overall transfer function can be calculated as the product of the transmittances of filtering (i.e., $T_{\text {filter }}$ ) and sensing (i.e., $T_{\text {sensor }}$ ) RRs as a function of $n_{c}$ (i.e., the refractive index of the cladding material in the sensible area), as reported in Eq. (1):

$$
T_{\text {tot }}=T_{\text {filter }} \times T_{\text {sensor }}\left(n_{c}\right)
$$

The filtering and sensing RRs are designed with different overall lengths (i.e., $L_{\text {filter }}$ and $L_{\text {sensor }}$, respectively) in order to exhibit two comb-like transfer functions char-

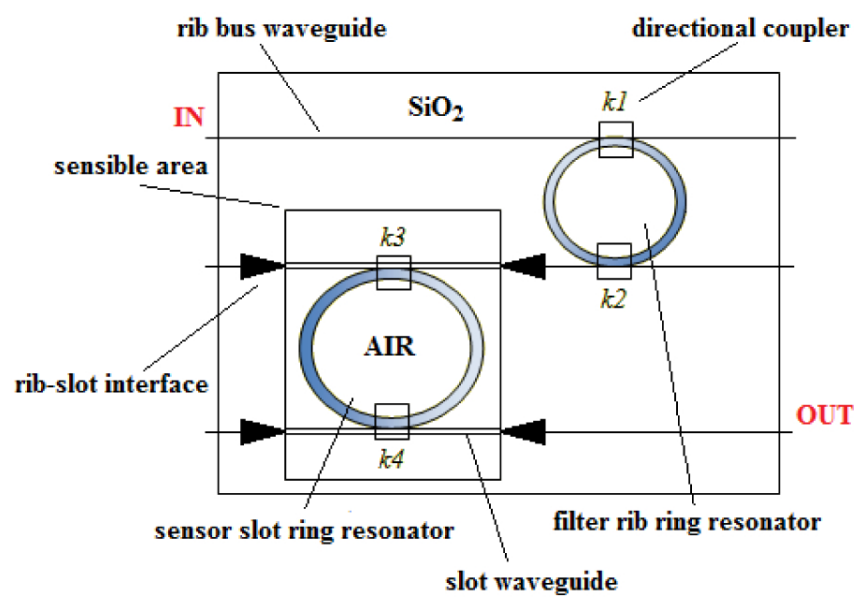

FIG. 1 Top view of the photonic sensor based on the Vernier effect.

acterized by different free spectral ranges (FSRs). In addition, such Vernier architectures can operate in the so called second operative Vernier regime, if the relationship $\triangle F S R<\Delta \lambda_{F W H M(\text { filter,sensor })}$ is satisfied. In particular, $\triangle F S R$ is the difference between filter and sensor FSRs (i.e., $\left.\Delta F S R=\left|F S R_{\text {filter }}-F S R_{\text {sensor }}\right|\right)$ and $\Delta \lambda_{F W H M(\text { filter,sensor })}$ indicates the smallest full-width at half maximum (FWHM) of resonant peaks of individual filtering and sensing RRs. When the overall sensor operates in the second regime of the Vernier effect, it is possible to demonstrate that sensing performance (i.e., wavelength sensitivity $S_{\lambda}=\frac{\Delta \lambda_{\text {tot }}}{\Delta n_{c}}$ and LOD) can be enhanced by a factor $G($ i.e., the Vernier gain) with respect to those achievable by a single RR characterized by analogous dimensions of the sensing RR constituting the Vernier architecture [15]. Moreover, the gain $G$ can be tuned by changing $L_{\text {filter }}$ and $L_{\text {sensor }}$, since it depends on both FSRs as reported in Eq. (2):

$$
G=\frac{F S R_{\text {filter }}}{\Delta F S R}
$$

Although spectral resonant peaks characterizing $T_{\text {filter }}$ are fixed because any refractive index perturbation cannot occur on the filtering RR silicon waveguide, resonant peaks of the sensing RR transfer function $T_{\text {sensor, }}$, can shift because of the interaction between the evanescent field of the optical mode propagating into the sensing RR waveguide and the test sample covering the windowed sensible area. In particular, a change of $n_{c}$ induces a wavelength shift of the Vernier spectrum according to Eq. (3):

$$
\Delta \lambda_{\text {tot }}=\Delta \lambda_{\text {sensor }} \times G
$$

where $\Delta \lambda_{\text {sensor }}=\lambda_{\text {sensor }} \times\left(\frac{\Delta n_{e f f}}{n_{e f f}}\right)$, being $n_{e f f}$ the effective index of the optical mode propagating into the sensing RR and $\Delta n_{e f f}$ the effective index change induced by the cover refractive index change (i.e., $\Delta n_{c}$ ) into the sensible area, which defines the waveguide homogeneous sensitivity indicated in Eq. (4):

$$
S_{h}=\frac{\Delta n_{e f f}}{\Delta n_{c}}
$$




\subsection{Design of silicon photonic waveguides for homogeneous sensing in mid infrared}

The main challenge in extending the operation of silicon Photonics from NIR to MIR wavelength region consists in the $\mathrm{SiO}_{2}$ high optical losses beyond $3.6 \mu \mathrm{m}$ wavelength [21], although silicon is a low loss material $\left(\alpha_{\text {loss }}<2 \mathrm{~dB} / \mathrm{cm}\right)$ for wavelengths up to $\sim 8 \mu \mathrm{m}$. Consequently, one possible criteria in the design of photonic devices operating at wavelengths longer than $3.6 \mu \mathrm{m}$, consists in limiting the amount of optical power confined into the $\mathrm{SiO}_{2}$ layers in order to reduce this propagation loss.

In case of Vernier architecture sketched in Figure 1, it is convenient to fabricate silicon waveguides able to confine an high percentage of the optical signal into the high refractive index material (i.e., silicon), minimizing the amount of the optical power confined into the BOX and cladding material (i.e., $\mathrm{SiO}_{2}$ ). To this purpose, we have designed a silicon rib waveguide as sketched in Figure 2(a), for the fabrication of input/output buses and filtering RR. Such a waveguide exhibits propagation losses lower than $2 \mathrm{~dB} / \mathrm{cm}$, well acceptable for overall footprints of a few $\mathrm{mm}^{2}$.

The sensing RR is constituted by a silicon slot waveguide designed to operate at $3.8 \mu \mathrm{m}$, as sketched in Figure 2(b). In fact, slot waveguides are well-known to be suitable for sensing applications since they allow to confine the optical field in the low refractive index cladding (i.e., air) where the analyte or gas to be detected are concentrated, strongly enhancing the chemical-to-optical transduction. In particular, in this guiding structure $\mathrm{SiO}_{2}$ does not represent a critical constrain for the sensor operation, since the presence of a $180 \mathrm{~nm}$-thick silicon layer between the slot region and the BOX limits the propagation loss to about $2.6 \mathrm{~dB} / \mathrm{cm}$ with an optical confinement factor in $\mathrm{SiO}_{2}$ as low as $23 \%$ [18].

The spatial distributions of quasi-TE (i.e., transverse electric) polarized optical modes propagating into rib and slot waveguides have been calculated by the finite element method (FEM) [22] and plotted in Figure 2(a)-(b). Refractive indices of silicon and $\mathrm{SiO}_{2}$ have been calculated at $3.8 \mu \mathrm{m}$ through Sellmeier equations.

Silicon waveguides considered in this work are assumed to be fabricated on 6-inch SOI wafers with $400 \mathrm{~nm}$-thick silicon layer on $2 \mu \mathrm{m}$-thick buried oxide (BOX) layer, using conventional e-beam lithography and ICP etching [18]. In particular, the slot waveguide homogeneous sensitivity has been calculated according to Eq. (4) as $S_{h}=0.2$ [23].

A technological solution adopted in the design of the Vernier architecture consists in employing a suitable rib-slot mode converter, experimentally demonstrated by M. Nedeljkovic et al. [18] and theoretically presented by V. M. N. Passaro et al. [24] for strip-slot conversion in NIR. In particular, the schematic of the rib-slot interface is sketched in Figure 3.

In conclusion, the use of the rib-slot mode converter imposes the design of rib and slot waveguides whose dimensions are

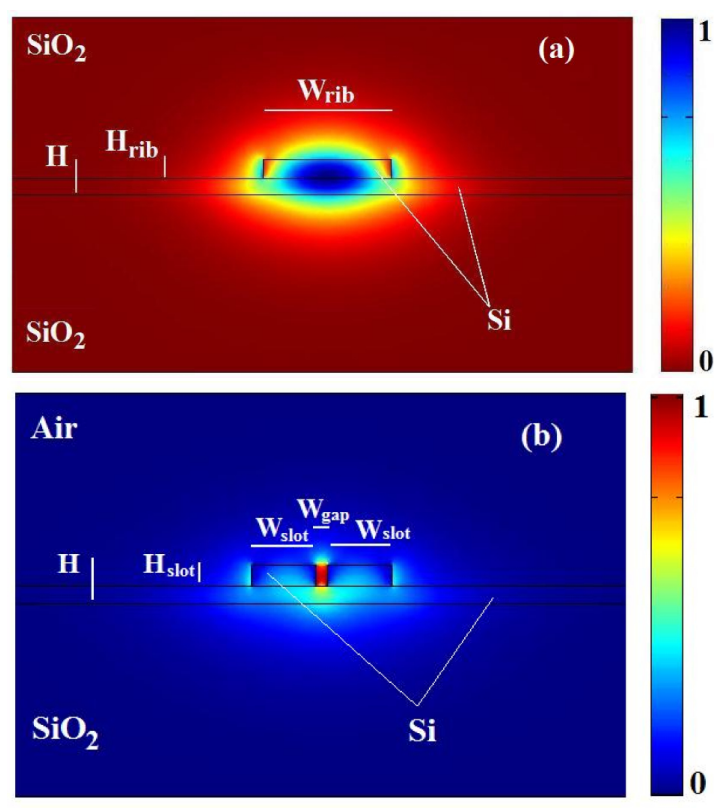

FIC. 2 Cross sectional views of SOI rib (a) and slot (b) waveguides with corresponding spatial distributions of quasi-TE optical modes propagating at $\lambda_{o p}=3.8 \mu \mathrm{m}$. Waveguide dimensions are: (a) $H=400 \mathrm{~nm}, H_{\text {rib }}=220 \mathrm{~nm}, W_{\text {rib }}=1440 \mathrm{~nm}$; (b) $H=400 \mathrm{~nm}, H_{\text {slot }}=220 \mathrm{~nm}, W_{\text {slot }}=660 \mathrm{~nm}, W_{\text {gap }}=120 \mathrm{~nm}$.

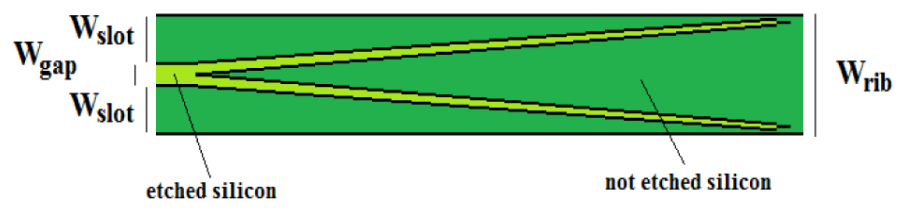

FIG. 3 Top view of the rib-slot mode converter operating at $3.8 \mu \mathrm{m}$.

characterized by the relationship $W_{\text {rib }}=2 \times W_{\text {slot }}+W_{\text {gap }}$. By using the rib-slot interface, slot waveguides as guiding structures for filtering RR and input/output buses are not needed, thus preventing high propagation loss due to the presence of $\mathrm{SiO}_{2}$ as cladding material.

\subsection{Directional coupler design}

The design of symmetrical directional couplers based on silicon rib and slot waveguides previously shown in Figures 2(a)(b), has been carried out by employing FEM, Coupled-Mode Theory (CMT) and Super-Mode Theory (SMT). Moreover, numerical results have been also validated by using the three dimensional (3D) semi-vectorial beam propagation method (SVBPM) [25]. Thus, design curves are plotted in Figures 4(a)-(b).

In Figure 4(a), the coupling length $L_{c}$ characterizing the directional coupler based on the silicon rib waveguide is plotted as a function of the directional coupler gap $g$. The theoretical approach consists in calculating a number of pairs $\left(L_{c}, g\right)$ labeled as "FEM-CMT discrete data" in the figure legends. Consequently, these data are processed in order to calculate an exponential design curve (i.e., FEM-CMT fitting data) as given in Eq. (5), where $A$ and $B$ are fitting parameters [26]:

$$
L_{c}=A e^{B g}
$$

In Figure 4(a), red points represent pairs $\left(L_{c}, g\right)$ calculated by 3D SV-BPM in order to validate the aforementioned theoreti- 

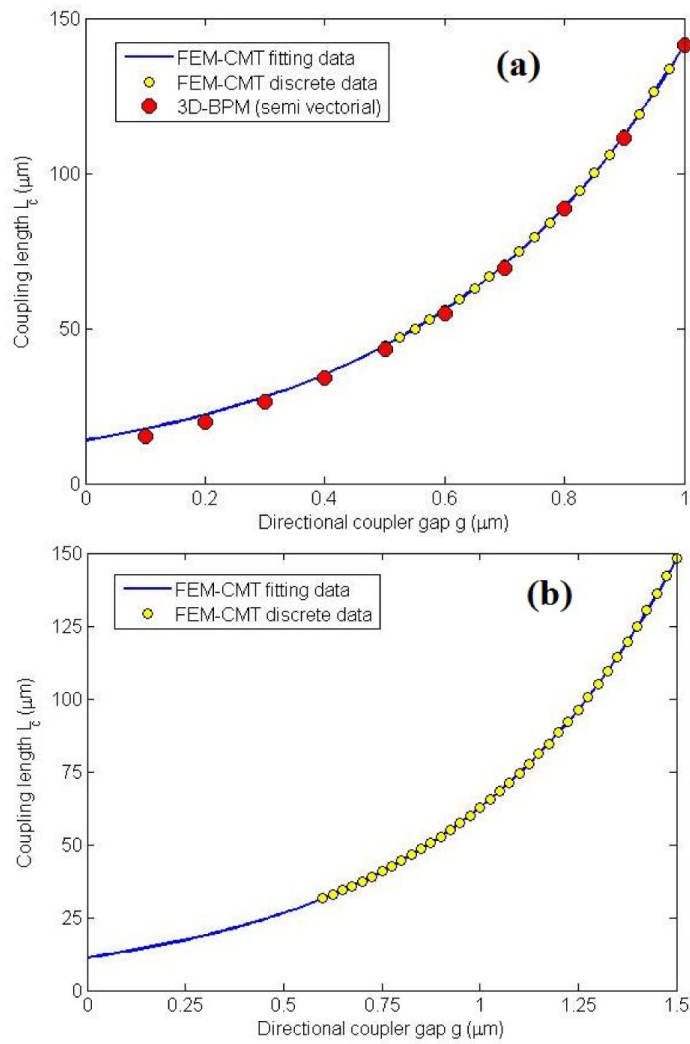

FIG. 4 Design of directional couplers based on silicon rib (a) and slot (b) waveguides operating at $3.8 \mu \mathrm{m}$.

cal approach. Numerical results evidence a very good agreement between the model based on CMT, SMT, FEM and 3D SV-BPM.

In conclusion, fitting parameters $A$ and $B$ and dimensions of directional couplers (i.e., $L_{c}$ and $g$ ) with corresponding power coupling coefficients $k_{i}$ are listed in Table 1 . It is to be noted that power coupling coefficients lower or equal to 0.1 are usually needed to maximize the amplitude spectrum of the overall Vernier transfer function [15].

\begin{tabular}{|l|l|l|}
\hline Parameters & $\begin{array}{l}\text { Directional } \\
\text { coupler (Rib) }\end{array}$ & $\begin{array}{l}\text { Directional } \\
\text { coupler (Slot) }\end{array}$ \\
\hline$A(\mu \mathrm{m})$ & 13.9386 & 11.2508 \\
\hline$B(1 / \mu \mathrm{m})$ & 2.3170 & 1.7167 \\
\hline$L_{c}(\mu \mathrm{m})$ & 100 & 70.25 \\
\hline$g(\mu \mathrm{m})$ & 0.510 & 0.7245 \\
\hline$k_{i}$ & $k_{1}=k_{2}=0.0953$ & $k_{3}=k_{4}=0.0953$ \\
\hline
\end{tabular}

TABLE 1 Characterization of directional couplers based on rib and slot waveguides at $3.8 \mu \mathrm{m}$ wavelength.

\section{OPERATION OF VERNIER RIB-SLOT PHOTONIC SENSOR OPERATING AT $3.8 \mu \mathrm{m}$}

A sophisticated algorithmic procedure has been implemented and used for the design of the Vernier architecture previously sketched in Figure 1 [15]. To this purpose, two sensor configurations characterized by different filtering and sensing RR lengths have been considered in our investigation, where the
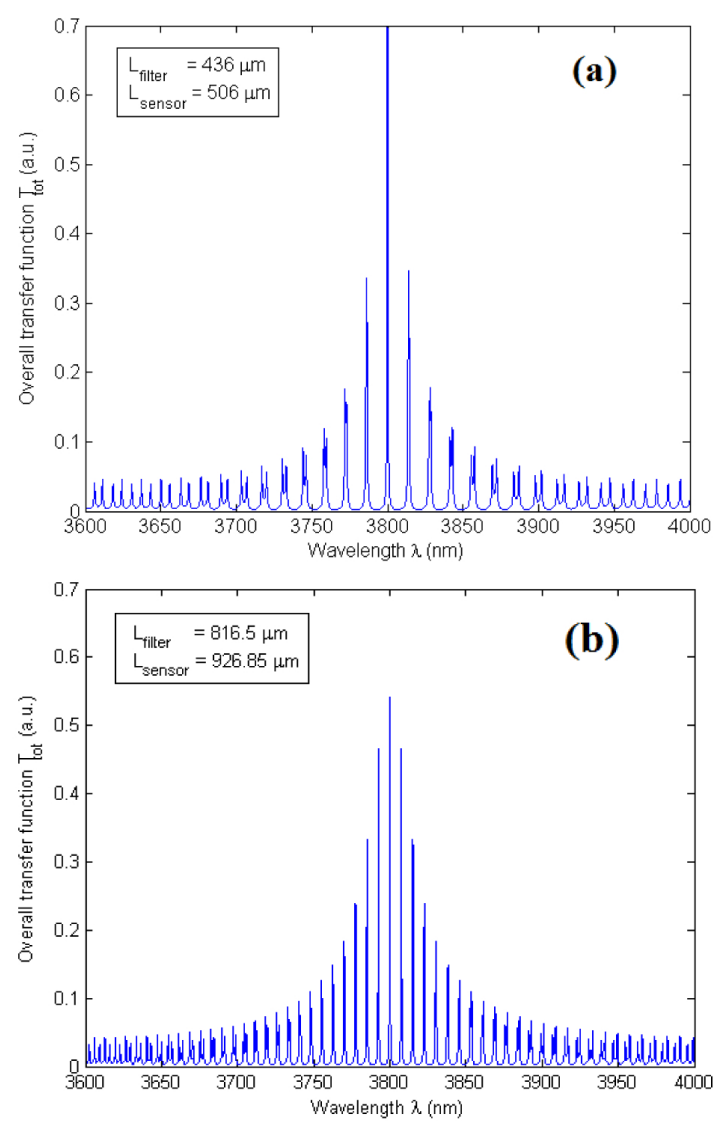

FIG. 5 Overall transfer functions of two Vernier sensor configurations (i.e., sensor \# 1 (a), sensor \#2 (b)), characterized by different filter and sensor RR lengths.

influence of propagation loss on sensor operation can be well appreciated being all other parameters fixed (i.e., directional couplers and corresponding power coupling coefficients $k_{i}$ as well as silicon rib and slot waveguides).

In both Figure 5(a)-(b), Vernier peaks are centered at the operative wavelength $\lambda_{o p}=3800 \mathrm{~nm}$, when sensors operate at rest, i.e. only air covers the slot waveguide into the sensible area. Furthermore, the amplitude of Vernier peaks $\left(T_{\text {peak }}\right)$ are different (i.e., $T_{\text {peak }}=0.7$ a.u. (a) and $T_{\text {peak }}=0.55$ a.u. (b)), being Vernier architectures characterized by different RR lengths. In particular, the longer $L_{\text {filter }}$ and $L_{\text {sensor, }}$ the lower the amplitude of Vernier peaks, since the effect of propagation loss is cumulated over longer distances. Moreover, Vernier peak amplitudes and overall spectral distributions also depend on directional coupler power coefficients.

In Table 2, sensing performance achievable by Vernier sensors proposed in this investigation are summarized, focusing on wavelength sensitivities $S_{\lambda}$, LODs (i.e., the minimum detectable cover refractive index change $\Delta n_{c, \min }$ induced by a cover refractive index change $\Delta n_{c, \text { min }}=5 \times 10^{-3} \mathrm{RIU}$ ) and Vernier gains $G$. In addition, we have compared these architectures with a similar one (i.e., sensor \#3) characterized by the sensing RR based on the rib waveguide designed at $3.8 \mu \mathrm{m}$ and sketched in Figure 2(a), instead of the silicon ribslot approach as analyzed previously. In particular, this sensor configuration is characterized by $L_{\text {filter }}=681.5 \mu \mathrm{m}$ and $L_{\text {sensor }}=733.05 \mu \mathrm{m}$ and is based on the same rib guiding structure for input/output buses as well as for filtering and 
sensing RRs. The rib waveguide covered by air into the sensible area exhibits an homogeneous sensitivity $S_{h}$ as low as 0.092 and a confinement factor of the optical power in $\mathrm{SiO}_{2}$ of $19.3 \%$. Finally, the identical directional couplers of the sensing RR are characterized by coupling lengths $L_{c} \approx 100 \mu \mathrm{m}$ and coupler gaps $g \approx 0.6 \mu \mathrm{m}$, exhibiting power coupling coefficients $k_{3}=k_{4}=0.0951$, quite similar to the coupling power coefficients listed in Table 1. Numerical results listed in Table 2 evidence the relationship among $\triangle F S R$ and sensing performance parameters.

\begin{tabular}{|l|l|l|l|}
\hline Parameters & Sensor \#1 & Sensor \#2 & Sensor \#3 \\
\hline$S_{\lambda}(\mu \mathrm{m} / \mathrm{RIU})$ & 9.2 & 20.6 & 7.553 \\
\hline$\Delta n_{c, \min }(\mathrm{RIU})$ & $1.54 \times 10^{-3}$ & $3.675 \times 10^{-4}$ & $1 \times 10^{-3}$ \\
\hline$G$ & 25.36 & 56.78 & 49.5 \\
\hline$\Delta$ FSR $(\mathrm{nm})$ & 0.5591 & 0.1333 & 0.1583 \\
\hline
\end{tabular}

TABLE 2 Sensing performance of three different Vernier sensor configurations operating in MIR.

In fact, by analyzing sensing performance of Vernier sensor $\# 1$ and sensor \#2, it is possible to observe that the lower the $\triangle F S R$ the higher the wavelength sensitivity and the Vernier gain, according to Eq. (2). On the contrary, LOD decreases by increasing $\triangle F S R$. In addition, the Vernier sensor \#3 exhibits a $\triangle F S R=0.1583$ similar to 0.1333 (i.e., $\triangle F S R$ of sensor \#2). In this way, although filtering and sensing RR lengths characterizing configurations sensor \#2 and sensor \#3 are different, their Vernier gains are quite similar.

The simulation of sensor \#2 operation is shown in Figure 6(a) and compared with that of sensor \#3, plotted in Figure 6(b). In particular, when a cover refractive index change $\Delta n_{c}=5 \times 10^{-3}$ RIU is assumed into the sensible area, an overall wavelength shift as long as $\Delta \lambda_{\text {tot }} \approx 100 \mathrm{~nm}$ occurs in sensor \#2. Moreover, the same cover refractive change $\Delta n_{c}$ is assumed into the sensible area of the Vernier configuration sensor \#3 and a wavelength shift $\Delta \lambda_{\text {tot }} \approx 40 \mathrm{~nm}$ occurs. This comparison evidences the importance of employing slot waveguides for the sensing RR fabrication, compensating the disadvantage of a more complex fabrication. In fact, slot waveguide homogeneous sensitivity is always higher than that achievable by a rib waveguide optimized for homogeneous sensing, resulting in a more efficient optical sensing. Consequently, by considering Vernier configurations sensor \#2 and sensor \#3, although they exhibit similar Vernier gains, sensor \#2 LOD is one order of magnitude lower than the sensor \#3 LOD and the wavelength sensitivity of sensor \#3 is approximately the half of $S_{\lambda}$ exhibited by sensor \#2.

\section{CONCLUSIONS}

The theoretical investigation on Vernier architectures operating at $3.8 \mu \mathrm{m}$, based on previous preliminary experimental results [17]-[20], confirms the concrete possibility to extend the operation of these photonic integrated chips in the MIR wavelength region, so opening novel scenarios for innovative photonic spectrometers and sensors. Ultra high sensing performance can be achieved by properly optimizing the design of the slot waveguide to be employed for homogeneous sens-
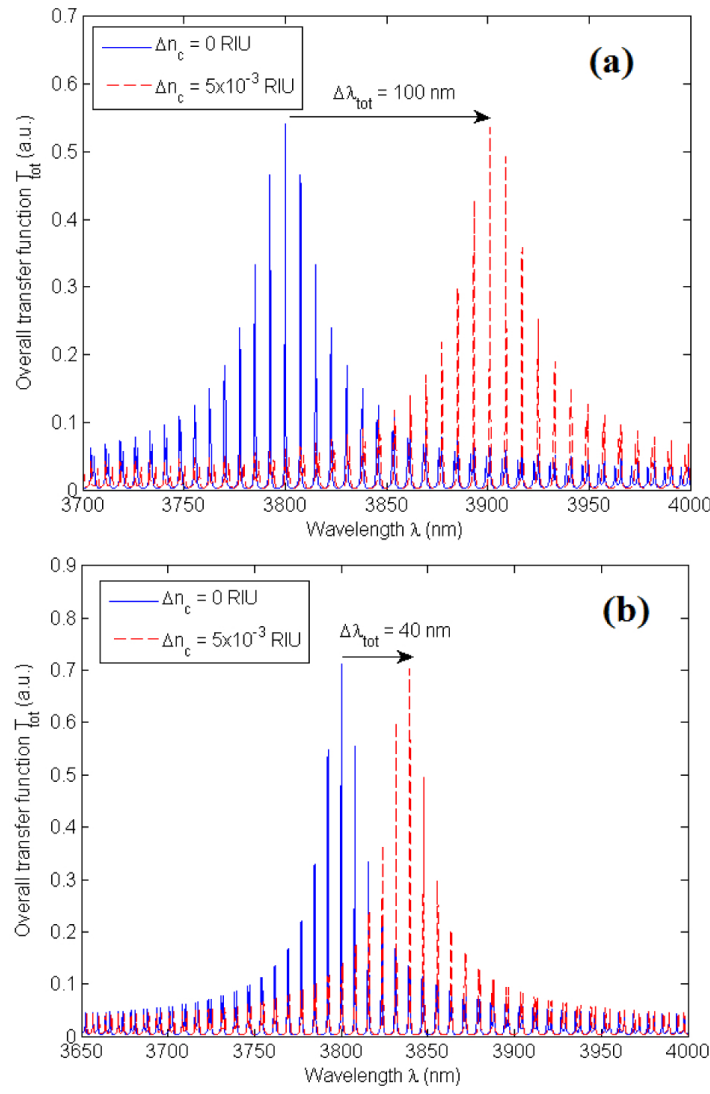

FIG. 6 Simulation of Vernier sensor \#2 (a) and sensor \#3 (b) operations.

ing in the sensible area and designing filter and sensor RR lengths in order to enhance the sensing performance as a function of the chemical specie to be detected. Finally, wavelength shifts characterizing the Vernier sensor operation can be easily detected by monitoring the envelope of the whole Vernier spectral distribution, resulting in a simple optical readout.

\section{References}

[1] L. Chrostowski, S. Grist, J. Flueckiger, W. Shi, X. Wang, E. Ouellet, H. Yun, et al., "Silicon photonic resonator sensors and devices," Proc. of SPIE, 8236, 823620 (2012).

[2] V. M. N. Passaro, B. Troia, M. La Notte, and F. De Leonardis, "Photonic resonant microcavities for chemical and biochemical sensing," RSC Adv. 3, 25-44 (2013).

[3] N. A. Yebo, S. P. Sree, E. Levrau, C. Detavernier, Z. Hens, J. A. Martens, and R. Baets, "Selective and reversible ammonia gas detection with nanoporous film functionalized silicon photonic micro-ring resonator," Opt. Express 20, 11855-11862 (2012).

[4] J. Hodgkinson, and R. P. Tatam, "Optical gas sensing: a review," Meas. Sci. Technol. 24, 1-59 (2013).

[5] M. Piliarik, H. Sipova, P. Kvasnicka, N. Galler, J. R. Krenn, and J. Homola, "High-resolution biosensor based on localized surface plasmons," Opt. Express 20, 672-680 (2012).

[6] M. Lee, and P. M. Fauchet, "Two-dimension silicon photonic crystal based biosensing platform for protein detection," Opt. Express 15 , 4530-4535 (2007).

[7] W. Bogaerts, P. De Heyn, T. Van Vaerenbergh, K. De Vos, S. Kumar Selvaraja, T. Claes, P. Dumon, et al., "Silicon microring resonators," Laser \& Photon. Rev. 6, 47-73 (2012). 
[8] R. Boeck, N. A. F. Jaeger, N. Rouger, and L. Chrostowski, "Seriescoupled silicon racetrack resonators and the Vernier effect: theory and measurement," Opt. Express 18, 25151-25157 (2010).

[9] R. Boeck, J. Flueckiger, L. Chrostowski, and N. A. F. Jaeger, "Experimental performance of DWDM quadruple Vernier racetrack resonators," Opt. Express 21, 9103-9112 (2013).

[10] L. Jin, M. Li and J-J. He, "Highly-sensitive silicon-on-insulator sensor based on two cascade micro-ring resonators with Vernier effect," Opt. Commun. 284, 156-159 (2011).

[11] T. Claes, W. Bogaerts and P. Bienstman, “Experimental characterization of a silicon photonic biosensor consisting of two cascaded ring resonator based on the Vernier-effect and introducing of a curve fitting method for an improved detection limit," Opt. Express $18,22747-22761$ (2010).

[12] D. Dai, "Highly sensitive digital optical sensor based on cascaded high-Q ring-resonators," Opt. Express 17, 23817-23822 (2009).

[13] J. Hu, and D. Dai, "Cascaded-Ring Optical Sensor With Enhanced Sensitivity by Using Suspended Si-Nanowires," IEEE Photon. Technol. Lett. 23, 842-844 (2011).

[14] X. Jiang, J. Ye, J. Zou, M. Li, and J.-J. He, "Cascaded siliconon-insulator double-ring sensors operating in high-sensitivity transverse-magnetic mode," Opt. Letters 38, 1349-1351 (2013).

[15] V. M. N. Passaro, B. Troia, and F. De Leonardis, "A generalized approach for design of photonic gas sensors based on Vernier-effect in mid-IR," Sens. Actuators B: Chemical 168, 402-420 (2012).

[16] B. Troia, and V. M. N. Passaro, "Photonic sensor based on the Vernier effect operating at $3.8 \mu \mathrm{m}$," in Proceedings of the $5^{\text {th }}$ EOS

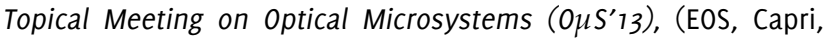
2013).
[17] G. Z. Mashanovich, M. M. Milosevic, M. Nedeljkovic, N. Owens, B. Xiong, E. J. Teo, and Y. Hu, "Low loss silicon waveguides for the mid-infrared," Opt. Express 19, 7112-7119 (2011).

[18] M. Nedeljkovic, A. Z. Khokhar, Y. Hu, X. Chen, J. Soler Penades, S. Stankovic, H. M. H. Chong, et al., "Silicon photonic devices and platforms for the mid-infrared," Opt. Mat. Express 3, 1205-1214 (2013).

[19] M. Muneeb, X. Chen, P. Verheyen, G. Lepage, S. Pathak, E. Ryckeboer, A. Malik, et al., "Demonstration of Silicon-on-Insulator midinfrared spectrometer operating at $3.8 \mu \mathrm{m}$," Opt. Express 21, 11659-11669 (2013).

[20] G. Roelkens, U. Dave, A. Gassenq, N. Hattasan, C. Hu, B. Kuyken, F. Leo, et al., "Silicon-based heterogeneous photonic integrated circuits for the mid-infrared," Opt. Mat. Express 3, 1523-1536 (2013).

[21] R. A. Soref, S. J. Emelett, and W. R. Buchwald, "Silicon waveguided components for the long-wave infrared region," J. Opt. A: Pure Appl. 0pt. 8, 840-848 (2006).

[22] Comsol Multiphysics by COMSOL $^{\odot}$, ver. 3.2, single license (2005).

[23] F. Dell'olio, and V. M. N. Passaro, "Optical sensing by optimized silicon slot waveguide," Opt. Express 15, 4977-4993 (2007).

[24] V. M. N. Passaro, and M. La Notte, “Optimizing Sol Slot Waveguide Fabrication Tolerances and Strip-Slot Coupling for Very Efficient Optical Sensing," Sensors 12, 2436-2455 (2012).

[25] OptiFDTD, Version 7.1 at OptiBPM, Version 9.0; Optiwave Systems Inc.: Nepan, 0N, Canada, 2007.

[26] V. M. N. Passaro, F. Dell’0lio, B. Timotijevic, G. Z. Mashanovich, and G. T. Reed, "Polarization-Insensitive Directional Couplers Based on Sol Wire Waveguides," The Open Optics Journal 2, 6-9 (2008). 